\title{
Spatial Biases and the Haptic Experience of Surface Orientation
}

\author{
Frank H. Durgin and Zhi Li \\ Swarthmore College, \\ USA
}

\section{Introduction}

The two main purposes of this chapter are to review past evidence for a systematic spatial bias in the perception of surface orientation (geographical slant), and to report two new experiments documenting this bias in the manual haptic system. Orientation is a fundamental perceptual property of surfaces that is relevant both for planning and implementing actions. Geographical slant refers to the orientation (inclination or pitch along its main axis) of a surface relative to the gravitationally-defined horizontal. It has long been known that hills appear visually steeper than they are (e.g., Ross, 1974). Only recently has it been documented that (1) there is also bias in the haptic perception of surface orientation (Hajnal et al., 2011), and that (2) similar visual and haptic biases even exist for small surfaces within reach (Durgin, Li \& Hajnal, 2010).

To provide a context for understanding the present experiments, we will first provide an overview of the prior experimental evidence concerning bias in the perception of geographical slant. First we will discuss findings from both vision and haptic perception that have documented perceptual bias for surfaces in reach. We will then review the literature on the visual and haptic biases in the perception of the greographical slant of locomotor surfaces such as hills and ramps. At the intersection of these two literatures is the historical use of haptic measures of perceived geographical slant, and we will therefore review these measures with particular attention to understanding some pitfalls in the use of haptic measures of perception. We next contrast these haptic measures with proprioceptive measures of perceived orientation and discuss the problem in interpreting calibrated actions as measures of perception.

Having laid out these various past findings we will then report two novel experiments that demonstrate spatial biases in the haptic experience of real surfaces. The experiments include both verbal and non-verbal methods modelled on similar findings we have reported in the visual domain. Following the presentation of the experimental results we will discuss issues of measurement in perception - especially pertaining to the interpretation of verbal reports, and conclude with a discussion of functional theories of perceptual bias in the perception of surface orientation.

\section{Spatial bias in the perception of orientation: Surfaces within manual reach}

What is meant by a spatial bias in the perception of surface orientation? Durgin, Li and Hajnal (2010) reported a series of studies of a bias they called the "vertical tendency" in slant 
perception. Specifically, they found that small, irregularly-shaped wooden surfaces appeared steeper than they actually were both when viewed visually and when experienced haptically while blindfolded. The term "vertical tendency" was used to distinguish the observed effect from what has been called "frontal tendency" in the literature (Gibson, 1950). For many years it has been argued that surfaces viewed visually, appear compressed along the depth axis of visual regard and thus appear more frontal to gaze than they are. However, when Durgin, Li and Hajnal asked participants to make estimates of the geographical slants of wooden surfaces within reach, they found that that they got approximately the same bias function whether the surfaces were at eye level (so that "frontal" and vertical coincided) or viewed with gaze declined by about $40^{\circ}$. Moreover, the same kinds of bias were found when surfaces were experienced haptically by placing the palm of the hand on them, though their measurements of this were limited to the angle of 0 $45^{\circ}$. The typical bias function for vision is shown in Figure 1.

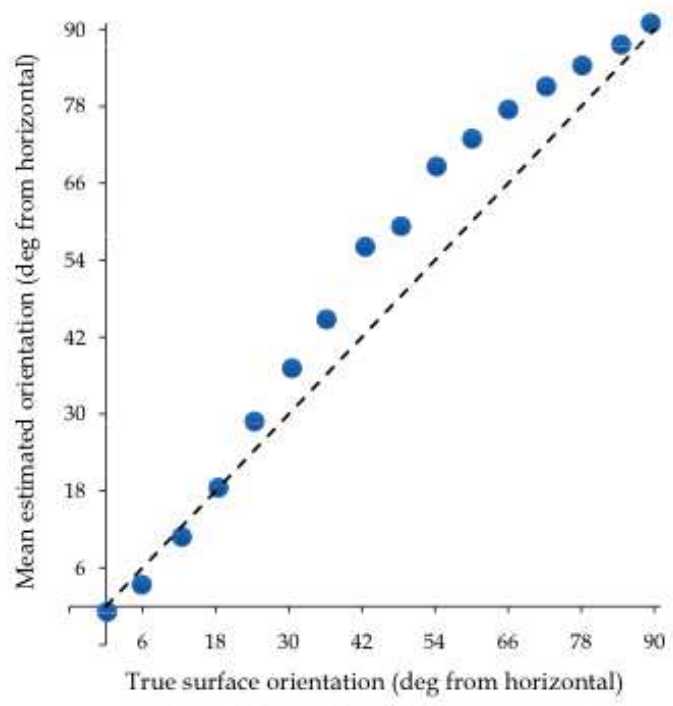

Fig. 1. Surface orientation estimates for near visual surfaces presented within reach of the hand (Durgin, Li \& Hajnal, 2010, Experiment 1). Symbol size approximates SEM.

The estimates shown in Figure 1 are based on verbal/numeric estimates of orientation relative to horizontal, but the bias observed cannot be due to verbal coding. Essentially the same spatial function was found if participants instead estimated orientation relative to vertical and their responses were then subtracted from $90^{\circ}$ in order to express them relative to horizontal. Thus for example, a surface that was actually at a $42^{\circ}$ orientation from horizontal (and thus $48^{\circ}$ from vertical), was estimated as being about $60^{\circ}$ from horizontal by one group of participants and about $30^{\circ}$ from vertical by another. Clearly both groups saw it as much steeper than its actual slant. When the same $42^{\circ}$ surface was explored, haptically, by a third group of participants by each placing the palm of the right hand against it while blindfolded, it was also judged to be $60^{\circ}$ from horizontal (Durgin, Li \& Hajnal, 2010, Experiment 4). 
Moreover, to emphasize that these biases did not depend on generating verbal estimates, Durgin, Li and Hajnal (2010) asked a fourth set of participants to judge whether various oriented planar surfaces were closer to horizontal or to vertical. They fit a psychometric function to the resulting choice data and found that a surface slanted by only $34.3^{\circ}$ from horizontal was, on average, visually perceived to be equidistant from vertical and horizontal.

This spatial bias function for near surfaces closely matches the observed proprioceptive function for the perceived declination of gaze. That is, when people are asked to report the pitch of their gaze, verbal reports provide evidence of an exaggerated deviation from horizontal that closely matches the bias function shown above for perceived surface slant (Durgin \& Li, 2011a; Li \& Durgin, 2009). Thus, it appears that several different perceptual representations of pitch contain a bias that expands the scale of differences near horizontal while compressing the scale near vertical.

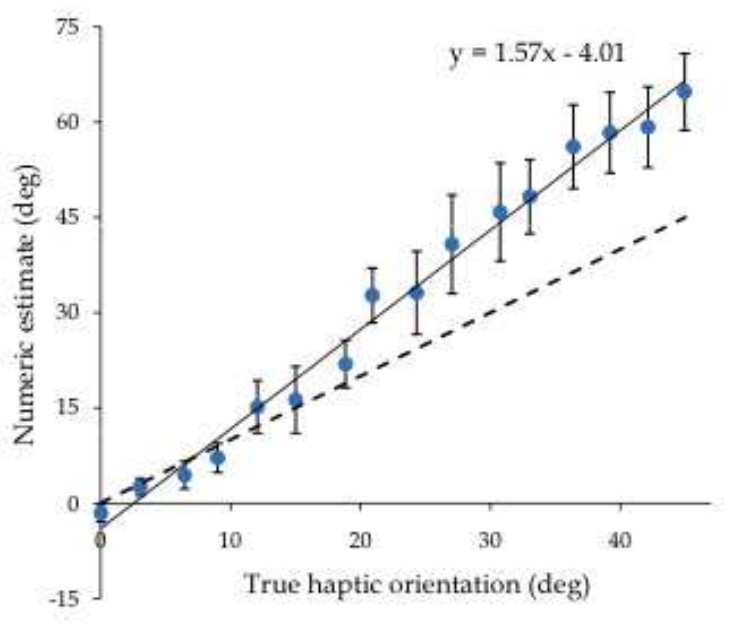

Fig. 2. Surface orientation estimates for surfaces $\left(0-48^{\circ}\right)$ felt with the palm of the hand (Durgin, Li \& Hajnal, 2010, Experiment 4). Error bars indicate \pm 1 SEM.

In the haptic domain and in the proprioception of gaze, the perceptual scale of the bias function for perceived pitch has mostly only been measured for orientations within about $50^{\circ}$ from horizontal (e.g., Durgin, Li \& Hajnal, 2010). In this range the scaling of pitch tends to closely approximate a linear scale with a gain of 1.5 (Durgin \& Li, 2011a). For example the haptic data of Durgin, Li and Hajnal are shown in Figure 2. These data are based on numeric estimates of orientation in deg (relative to horizontal) made based on placing the palm of the hand on various real slanted surfaces while blindfolded. Durgin and $\mathrm{Li}$ have reported a very similar function for explicit estimates of the pitch of gaze over a similar range. Durgin and Li (like Durgin, Li \& Hajnal) supplemented their verbal estimation data with a horizontal-vertical bisection task and again found that a rather shallow gaze declination of about $30^{\circ}$ from horizontal was perceived as the bisection point between vertical and horizontal gaze. 
These observations are particularly relevant to the present discussion because they emphasize that the form of the perceptual bias may not be a "vertical tendency." Rather, the linear gain of about 1.5 found in various measurements of perceived pitch between $0^{\circ}$ and $45^{\circ}$ from horizontal suggests that deviations from horizontal are being exaggerated and therefore that it is the horizontal that is special. Durgin and Li (2011a) have hypothesized that the expanded scaling (near horizontal) of the perceived pitch of gaze direction serves to expand the most highly utilized portion of the angular range for purpose of maintaining greater cognitive precision. Across a variety of contexts, they proposed that a gain of about 1.5 may provide efficient scaling for retaining greater precision in this lower half of the range when sending neural pitch signals upstream to cognitive and motor areas.

\section{Bias in the perceived orientation of locomotor surfaces: Hills and ramps}

While there are clear and consistent visual and spatial biases in the perception of surfaces within reach of the hands, visual biases measured in the perception of locomotor surfaces have tended to be seem even more exaggerated. These exaggerations were initially documented by Kammann (1967) who proposed that they might reflect the heightened energetics of the gravitational vertical, and by Ross (1974), but were most extensively documented by Proffitt et al. (1995). Although many of the theoretical conclusions of Proffitt et al. have been called into question by more recent work, their basic documentation of the judged orientation of 8 distinct hills on their campus remains a useful basic source regarding the overestimation of hills. In particular, Proffitt et al. reported that paths with physical slants of $4^{\circ}, 5^{\circ}$ or $6^{\circ}$ were typically perceived as being about $20^{\circ}$ in orientation when viewed with gaze forward, as shown in Figure 3.

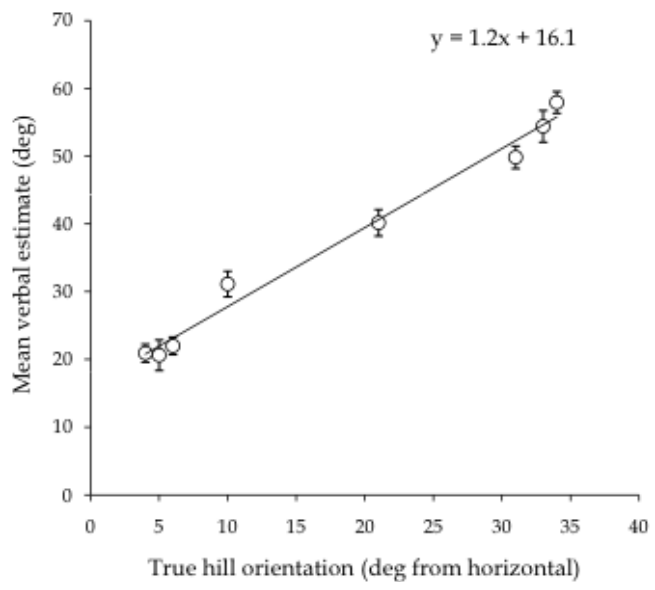

Fig. 3. Verbal estimates of the (visual) slants of eight hills from Proffitt et al. (1995), \pm 1 SEM. Hills were viewed from the base with gaze forward.

Because the range of orientations in most locomotor settings is no more than $\pm 10^{\circ}$ from horizontal, and physical/biomechanical limitations restrict bipedal locomotion to surfaces of less than about $35^{\circ}$, Proffitt et al. (1995) proposed that the exaggerated perception of hill orientation was an adaptive strategy to represent the range of human behavioural potential 
and energetic costs and thus to inform decisions about route selection during locomotion in the wild. A difficulty with this view is that perceived hill orientation decreases as one approaches a hill (Li \& Durgin, 2010), and nearer portions of hills appear shallower than farther portions (Bridgeman \& Hoover, 2008). In fact, as we will discuss below, there is continuity between orientation biases we have measured for small, near surfaces and those measured by Proffitt et al. for hills (Li \& Durgin, 2010).

Proffitt further proposed that physical actions, such as stepping, were controlled by an unbiased perceptual representation (vision for action) that was contrasted with the exaggerated representation available for long-range cognitive planning. This view has since been challenged by studies of the haptic perception of locomotor inclines.

\subsection{Haptic bias in the perceived orientation of locomotor surfaces}

Hajnal et al. (2011) asked participants to step onto ramps that they could not see. (They were either wearing a blindfold or an occluding collar that blocked their view of the floor.) The ramps varied in orientation from $4^{\circ}$ to $16^{\circ}$. Participants were asked to provide either verbal estimates of the surface orientation or to gesture the orientation with the their hand, which was measured using digital photography. The data are reproduced in Figure 4, along with a photograph of the experimental situation. Both forms of measurement documented surprisingly-large perceptual exaggerations of haptic slant. For somewhat steep ramps, the haptic exaggeration of perceived slant was even greater than the visual exaggeration observed when the same participants judged the orientations of the ramps when looking at them afterward. For example, Hajnal et al. found that participants standing on a $16^{\circ}$ ramp judged it to be about $35-40^{\circ}$ (both verbally, and as measured by hand gesture) based on their haptic experience, whereas when looking down at a $16^{\circ}$ ramp (while standing on a level surface at the base of the ramp) they judged it to be only about $22-24^{\circ}$. The same pattern (higher estimates based on haptic perception) was found for a $14.5^{\circ}$ ramp by Durgin et al. (2009) who collected visual estimates before having people step onto the ramp.

Haptic evaluations of the surface under one's feet are more valuable for immediate motor planning than for distal route planning. Hajnal et al. (2011) therefore suggested that these distortions might be the perceptual consequence of dense coding of orientations near horizontal that led to functionally exaggerated perceived orientation for the more precise control of action.

Kinsella-Shaw et al. (1992) had previously reported that participants were good at matching haptic inclines underfoot to visual inclines. To rule out the possibility that the haptic exaggerations were learned from calibrating haptic experience to visual experience, Hajnal et al. (2011) also tested a population of four congenitally blind individuals using verbal report. The blind individuals' estimates were quite similar to those of the sighted participants, though they were slightly higher. This indicates that the haptic exaggeration of the apparent inclination of surfaces on which one stands exists even in the absence of visual experience.

\subsection{Proprioceptive bias in the perceived orientation of locomotor surfaces}

Proprioceptive error in the perceived declination of gaze was first reported in a study of downhill slant perception: Li and Durgin (2009) observed that standing back from the edge of an outdoor downhill surface made it appear steeper than when standing closer to the 
edge. Indeed, for a steep hill, the maximum perceived orientation seemed to occur when standing far enough back from the edge of the hill that one's line of gaze was nearly coincident with the surface of the hill. Using a virtual environment in which viewing position was manipulated orthogonally to the steepness of the incline, $\mathrm{Li}$ and Durgin found that the functions relating simulated optical slant to perceived optical slant only lined up with one another at the two viewpoints if it was assumed that the change in perceived declination of gaze was exaggerated with a gain of about 1.5 .
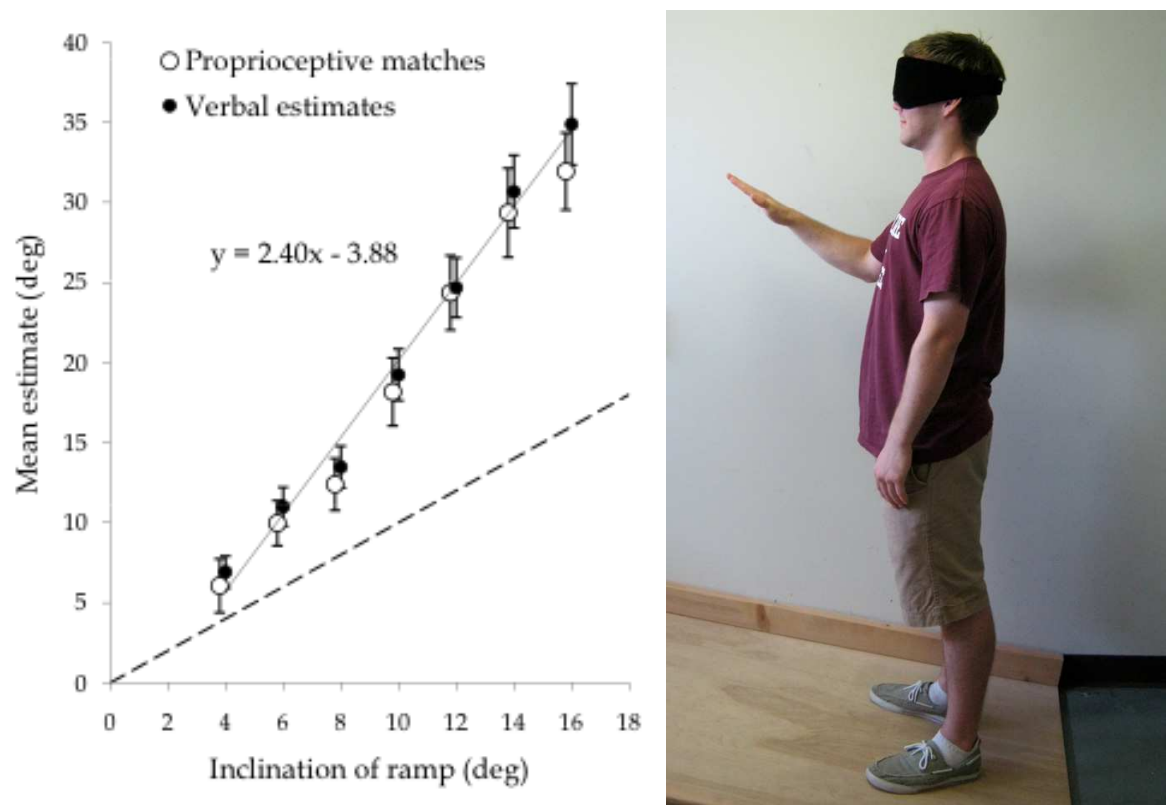

Fig. 4. Verbal and proprioceptive (hand gesture) estimates of the haptically-perceived orientation of a ramp while standing on it, blindfolded (from Hajnal et al., 2011). Hand gesture data has been recomputed to represent the main orientation of the hand rather than the orientation of the palm, which is about $6.5^{\circ}$ steeper (see Durgin, Li \& Hajnal, 2010). Proprioceptive points are displaced to show the SEMs. Fit line is to verbal data.

Deducing from these observations that the perceived direction of gaze might itself be distorted Li and Durgin (2009) tested this directly by asking people to look at targets at various declinations out of upper-floor windows and estimate the downward pitch of their gaze. Again, a gain of 1.5 was found. Later studies confirmed that the gain of perceived gaze declination is about 1.5 even for objects in near space and along a locomotor surface (Durgin et al., 2011), as discussed in Section 2.

\subsection{Continuity between visual biases for near and far surfaces}

Across a number of studies, Li and Durgin (2009, 2010; Durgin \& Li, 2011a) have found systematic evidence suggesting that the visual perception of slant also has a gain of $1.5 \mathrm{in}$ the low end of the geographical slant range. But this led to a puzzle. If perceived slant has a 
gain of only 1.5 , then why does a hill of $5^{\circ}$ appear to be $20^{\circ}$ rather than, say, $7.5^{\circ}$ ? And why is the perceptual gain for outdoor hills, as illustrated in Figure 3, limited to a factor of about 1.2? Following up on the observations of Bridgeman and Hoover (2008), Li and Durgin (2010) noted that viewing outdoor hills from the base of the hill with gaze forward means that geographical slant is confounded with viewing distance to the hill surface. For example, for a typical eye-height of $1.6 \mathrm{~m}$, a $5^{\circ}$ hill is viewed at a horizontal distance of $18 \mathrm{~m}$, whereas a $30^{\circ}$ embankment would be viewed at a horizontal distance of less than $3 \mathrm{~m}$.

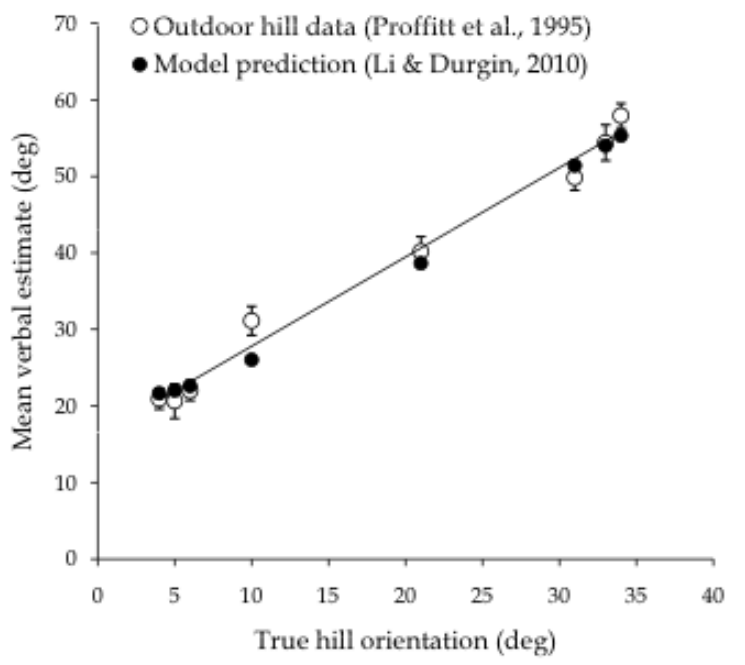

Fig. 5. Predictions of outdoor visual data from Proffitt et al. (1995) based on a model with only one free parameter. With the overall intercept set to $0^{\circ}$, and the slant gain fixed at 1.5, the perceived slant model depicted here is simply $\beta_{p}=1.5 \cdot \beta+k \log (D)$, where $D$ denotes the horizontal viewing distance from the base of the hill (assuming an eye height of $1.6 \mathrm{~m}$ ), $\beta$ denotes actual slant and $\beta_{p}$ denotes perceived slant; the one free parameter, $k$, equals 5 .

Using a high quality virtual environment, Li and Durgin (2010) decoupled viewing distance and geographical slant by presenting large full-cue binocular surfaces at 6 orientations in the linear range of $6-36^{\circ}$ at 5 distances in the logarithmic range of 1 to $16 \mathrm{~m}$. Viewing was horizontal. They found that at each viewing distance the gain of perceived slant was about 1.5, but that the intercept of the slant function increased with the log of distance. Thus, the 30 combinations of orientation and distance could be fit with a three-parameter model including an overall intercept, a constant multiplied by log distance and a slant gain of about 1.5). Despite the many potential differences between virtual environments and real ones, $\mathrm{Li}$ and Durgin showed that a 3-parameter model derived from their verbal report data in the virtual environment provided an excellent fit to Proffitt et al.'s (1995) outdoor hill data when the viewing distance required for each hill was taken into account. By setting the slant gain to 1.5 and assuming an overall intercept of $0^{\circ}$, we can reduce the model to a single free parameter, based on the multiplier of log viewing distance. The predictions of such a model are shown in Figure 5 alongside each of the observed mean estimates from Proffitt et al. The success of this model in fitting the outdoor data shows that, once viewing distance is taken into account, the underlying slant gain for outdoor hills as for small surfaces seems to be 1.5. 
This analysis provided by Li and Durgin (2010) shows how the apparent discrepancy between the perceived slants of hills and of near surfaces may be due to differences in viewing distance. However, the model does not explain why haptic slant perception of ramps underfoot has such a high gain. The most intriguing observation we can make about this concerns the discrepancy between the haptically perceived slant of the $16^{\circ} \operatorname{ramp}\left(\sim 35^{\circ}\right)$ and the visually perceived slant of that same ramp $\left(\sim 23^{\circ}\right)$. Because the ramp was viewed at a near viewing distance, with head declined, the resulting exaggerated scaling in vision ought to be by about 1.5 times, and it was. In contrast, if a $16^{\circ}$ hill were viewed with gaze forward, the horizontal distance to the surface would be $5.6 \mathrm{~m}$ away, and the model prediction would be a perceived slant of $32.6^{\circ}$, which is quite close to the haptically-perceived slant of the $16^{\circ}$ ramp. In contrast, for a $6^{\circ}$ ramp, the estimates given haptically and from visual estimates of the ramp were in close agreement with one another $\left(\sim 11^{\circ}\right.$, Hajnal et al., 2011$)$. Although these were both far lower than (i.e., about half) what would be expected for forward viewing of a $6^{\circ}$ hill, a value of $\sim 11^{\circ}$ is consistent with predictions of the one-parameter model for the actual viewing distance of about $1.8 \mathrm{~m}$. Thus, the data of Hajnal et al. suggest that there is indeed some calibration between pedal and visual estimates of slant for common slants (of $10^{\circ}$ or less) of near surfaces, as Kinsella-Shaw et al. (1992) suggested. However, Hajnal et al. (2011) have emphasized that the biomechanics of placing the foot upon a locomotor surface allow for rapid accommodation of the foot to the surface and may not require a very precise visual estimate of surface orientation in order for stepping to be successful. It is probably surprising to many that using hand gestures to try to match the slant of the surface on which one stands produces as much error as it does. This seems strong confirmation that the perceptual experience of the slants underfoot really is quite exaggerated. Because of the limited range of upward flexion of the foot, the extreme scaling of pedal slant is consistent with the idea of sensory scaling of perceived ramp orientation partly representing the biomechanical range of flexion. The evidence that a similar magnitude of perceptual exaggeration is present in participants who are congenitally blind lends support to this interpretation, by indicating that calibration is not the source of the haptic distortion. It seems unlikely that the visual distortion derives from the haptic.

\section{Problems with measuring perceived slant with haptic matching tasks}

One current controversy in the study of slant perception concerns a popular method of assessing perceived slant. Proffitt et al. (1995) developed a method of assessing perceived slant that they initially referred to as a haptic measure, but also (e.g., Bhalla \& Proffitt, 1999) described as an action measure. The measure consists of using one's hand to adjust the orientation of a "palm board" so as to match the perceived slant of a surface. The palm board was originally developed by Gibson (1950) as a non-verbal measure of perceived slant. In their studies of hills, Proffitt et al. (1995) placed the palm board at waist level so that it was at the edge of the field of view of the observer. They found that unlike verbal measures, which overestimated hill orientations, the palm board measure was relatively accurate. Bhalla and Proffitt interpreted the relative accuracy of the palm board measure as evidence of an accurate underlying perceptual representation "for action." However, some simple control experiments carried out by Durgin, Hajnal, Li, Tonge and Stigliani (2010) suggested that that palm boards were only accidentally accurate.

Durgin, Hajnal, Li, Tonge and Stigliani (2010) reasoned that if palm boards were assessing accurate motor representations of space, then they ought to be particularly accurate for 
matching near surfaces with which the hand could actually interact. That is, Durgin et al. presented full-cue wooden surfaces within reach and had people try to match their orientations using a palm board. Rather than being accurate, as the action theory predicted, palm board settings were much too low. Durgin et al. interpreted this as a haptic/proprioceptive error due to inaccurate scaling of wrist flexion. Durgin et al. showed that people overestimated the flexion of their wrist with about the same gain as they overestimated far surfaces. Li and Durgin (2011a) showed that when verbal estimates of near surfaces (similar to those shown in Figure 1) were used to predict palm board matches to those surfaces the function relating the two measures was identical to the function that related verbal estimates of hills to palm board matches to those hills. In other words, the perceived orientation of the palm board was exaggerated in a way that (imperfectly) approximated the exaggeration of hills viewed at a distance. Palm board measures were not tapping into a separate motor representation, but rather were differently-scaled outputs tapping into the same distorted representation as verbal reports. When the distortion in vision was approximately cancelled by the distortions in proprioception/haptics, the illusion of accuracy resulted.

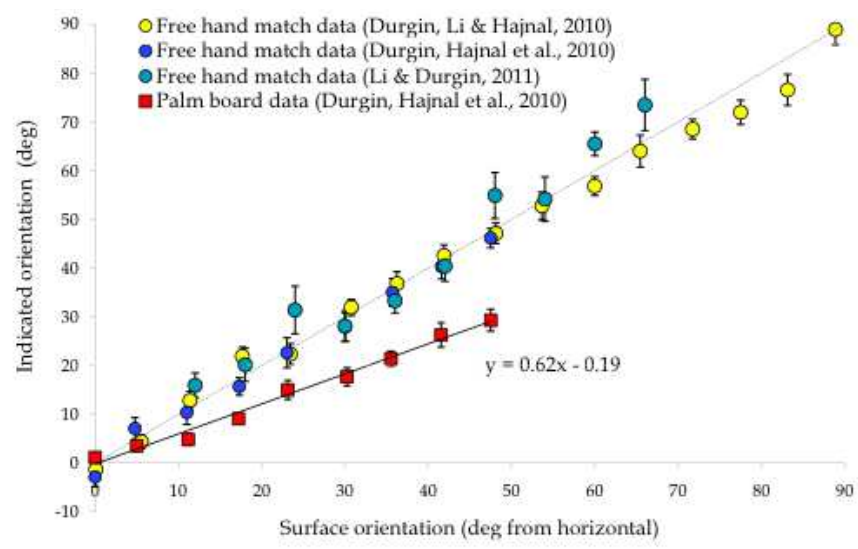

Fig. 6. Contrasting the gain of a palm board measure (i.e. 0.62) with the gain of a free-hand gesture for matching full-cue surfaces within reach (i.e., $\sim 1.0)$. The hand was occluded from vision in all cases. The visual surfaces were wooden surfaces within reach of the hand.

Strikingly, Durgin, Hajnal, Li, Tonge and Stigliani (2010, 2011) also showed that proprioceptive performance for near surfaces was greatly improved if the palm board were simply removed and people were allowed to gesture freely with their hand (with the hand hidden behind an occluding barrier). Some of their data are shown in Figure 6. As in the study of the haptic perception of ramps underfoot, free-hand gestures for far hills were found to grossly overestimate the slants of those hills (roughly consistent with verbal reports), but free-hand gestures for surfaces in near space were quite precise and accurate. The main difference between free-hand gestures and palm board matches were that palm boards prevented the use of the elbow as a primary axis of hand rotation. Because the axis of the palm board was near the wrist, the wrist had to be the principal joint for adjusting the palm board. Moreover, Durgin, Li and Hajnal (2010) showed that the perceived orientation of a fairly steep palm board was even higher than haptic perception of a rigid surface of the 
same orientation - perhaps because of the additional forces used to maintain the tilted orientation of the palm board.

In summary, haptic matching tasks have proven to be very difficult to interpret for at least three reasons. First, evidence of surprising accuracy between palm board matches and hills has turned out to be spurious. Palm boards simply feel much steeper than they are because they require flexing the wrist more than is customary in normal circumstances. Second, the perceptual gain of haptic surface perception is generally unknown. If people see a visual surface as $45^{\circ}$ when it is only $34^{\circ}$ and they feel that a haptic surface is $45^{\circ}$ when it is only $34^{\circ}$, they may seem to be correctly matching a $34^{\circ}$ surface when they think they are matching a $45^{\circ}$ surface. Finally, the fact that passive contact with a rigid surface dissociates from active rotation of a palm board suggests that haptic measures can be contaminated by active control of the surface's orientation.

\subsection{Calibration between proprioception and the visual experience of slant}

The function shown in Figure 6 for free-hand manual gestures was obtained with the same set of surfaces used to obtain the function in Figure 1 for verbal estimates of slant. Nonetheless the verbal estimates show a great deal of bias (exaggeration of the deviation from horizontal), whereas the manual gestures appear to be well-calibrated. One possibility is that proprioception is calibrated to vision. That is, the perceived orientation of the hand ought to match the perceived orientation of the surface. In support of this view, Li and Durgin (submitted) have found that perceived hand orientation during free-hand gestures follows the same function as the verbal pattern shown in Figure 1.

\subsection{An apparent discrepancy in the calibration account}

So far we have suggested that hand gestures are calibrated to near surfaces, but are not calibrated for far surfaces (which seem steeper). We have argued that palm board measures, which have been described by some as calibrated for hills, aren't really calibrated to visual surfaces at all. And we have suggested that the haptic experience of slants underfoot may be partly calibrated to the visual experience of hills, but needn't be. The guiding rule might be that calibration occurs when there is some real possibility for action with immediate spatial feedback from more than one modality. Underfoot surface calibration may be unnecessary because the foot is biomechanically adaptive and people tend not to look at their own feet when walking. Touching surfaces manually provides haptic and visual feedback.

However, there is one apparent exception to this proposed guiding rule. Hajnal et al. (2011) used a force feedback robotic arm (Phantom) to allow participants to feel a virtual surface. With this (carefully calibrated) device, they collected verbal estimates of perceived slant. Given the calibration account (based on the potential for shared visual and haptic experience in normal life) we should expect that haptic exploration of a surface by dynamic touch would reveal the same kind of spatial bias that is evident in vision (Figure 1) and in static haptic contact (Figure 2). In fact, although Hajnal et al. did observe overestimation of slant in their procedure, they applied a linear fit to their data that they interpreted as suggesting a fairly constant overestimation across all orientations. Their fit line is shown in the left panel of Figure 7. In fact, most of their data can be captured by a curved bias function such as we have seen in other data. As shown in the right panel of Figure 7, only 
the lowest three plot points deviate from the typical curvature we have observed elsewhere. We therefore sought to replicate their dynamic touch result. We chose to use a real physical surface instead of a virtual one.
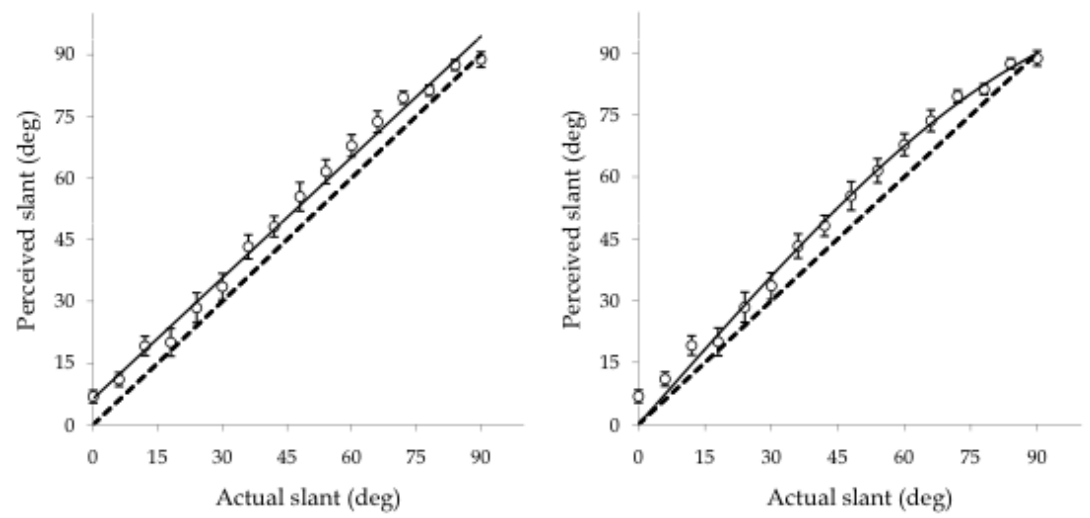

Fig. 7. Hajnal et al.'s (2011) dynamic touch data with two different fit lines. In the left panel, the linear fit line originally plotted by Hajnal et al. is shown. In the right panel, a cubic function with an intercept of $(0,0)$ was fit to the data.

\section{Experiment 1: Numeric estimation of real surface orientation in depth assessed by dynamic touch with the tip of the index finger}

The main question of the present experiment is whether the haptic perception of surface orientation (geographical slant in the pitch axis relative to the observer) by dynamic touch will show the same kinds of spatial bias documented in vision by Durgin, Li and Hajnal (2010). Whereas Durgin, Li and Hajnal reported evidence of similar bias in perceived surface orientation based on static contact with the palm of the hand, Hajnal et al. (2011) have argued that there is very little bias evident in dynamic touch. However, as noted above, it is not clear that the linear fit they plotted is better justified by their data than a cubic fit, like that shown in Figure 7. Moreover, examination of the raw data of Hajnal et al. suggested that participants relied nearly exclusively on angular estimates that were multiples of 5 . This may have contributed to distorting the lower end of the range. Finally, because Hajnal et al. did not constrain their participants' exploratory strategies, it is possible that the observed function was less exaggerated in some places because of a tendency for oblique paths of travel along the slanted surface. In the present study we used real surfaces and provided a ridge along the main axis of the surface to ensure that the steepest direction of inclination was felt. In addition we asked that participants be as precise as possible in their responses.

\subsection{Method}

All experimental procedures were conducted in accord with the ethical standards of the American Psychological Association and approved by a local institutional review board. The general method is similar to those employed by Hajnal et al. (2011) and by Durgin, Li and Hajnal (2010). Participants made numeric estimates of the slant of surfaces explored haptically. 


\subsubsection{Participants}

The participants were 20 Swarthmore College undergraduate students (13 female) who participated in partial fulfilment of a course requirement. Half were assigned to the horizontal coding condition and half to the vertical coding condition.

\subsubsection{Apparatus}

The haptic surface was a varnished wooden board mounted on a mechanical adjustable slant device (see Li \& Durgin, 2009). The center of the surface was about $113 \mathrm{~cm}$ from the floor. A metal ridge was attached to the surface perpendicular to the axis of rotation as a guide. Participants stood in front of the apparatus wearing a blindfold (a plush sleep mask) throughout the experiment. The set-up is shown in Figure 8.

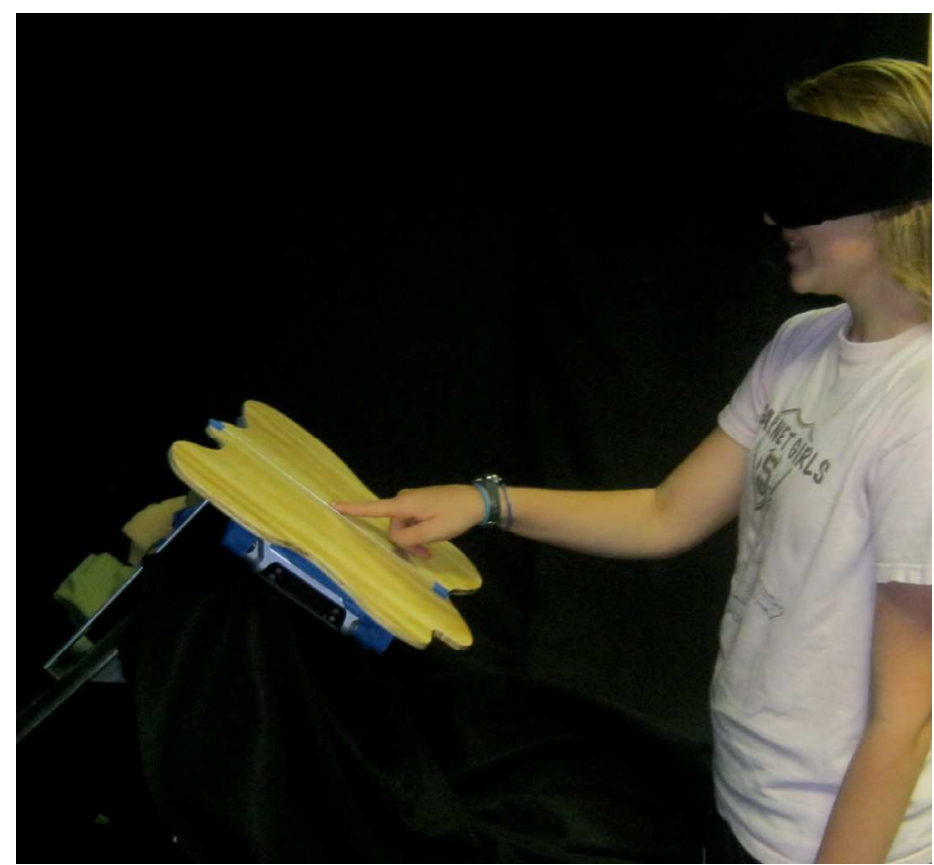

Fig. 8. The experimental set up. The orientation of the board could be rapidly adjusted to one of 16 orientations from $0^{\circ}$ to $90^{\circ}$.

\subsubsection{Design}

Participants were assigned in alternation to the vertical or horizontal coding condition. Following the practice of Durgin, Li and Hajnal (2010), half the participants gave verbal estimates relative to horizontal and half gave estimates relative to vertical so that spatial biases could be distinguished from verbal biases. In each condition the same set of 16 orientations from 0 to $90^{\circ}$ (by $6^{\circ}$ increments) were presented in random order in each of two blocks of trials for a total of 32 trials. Random orders were generated in advance for each participant. 


\subsubsection{Procedure}

Participants were shown the apparatus with the surface in the horizontal position and the procedure was explained to them prior to signing an informed consent form. Participants were shown where to stand (directly in front of the apparatus) and then asked put on the blindfold. Before each trial, the surfaces were set to the intended orientation manually using pre-set positions by the experimenter who then told the participant to explore the surface. The participants were to run the tip of their right index finger alongside the elevated ridge formed by a wire attached to the surface. No time limit was specified for exploration. When satisfied with their haptic observation the participant was to indicate the orientation of the surface in deg. Half were instructed that vertical was $0^{\circ}$ and horizontal was $90^{\circ}$. The other half were told to consider horizontal to be $0^{\circ}$ and vertical to be $90^{\circ}$. Participants were encouraged to be as precise as possible in their estimates by estimating orientation to the nearest $1^{\circ}$ (even with such instruction, there is a strong bias toward values divisible by 5 ).

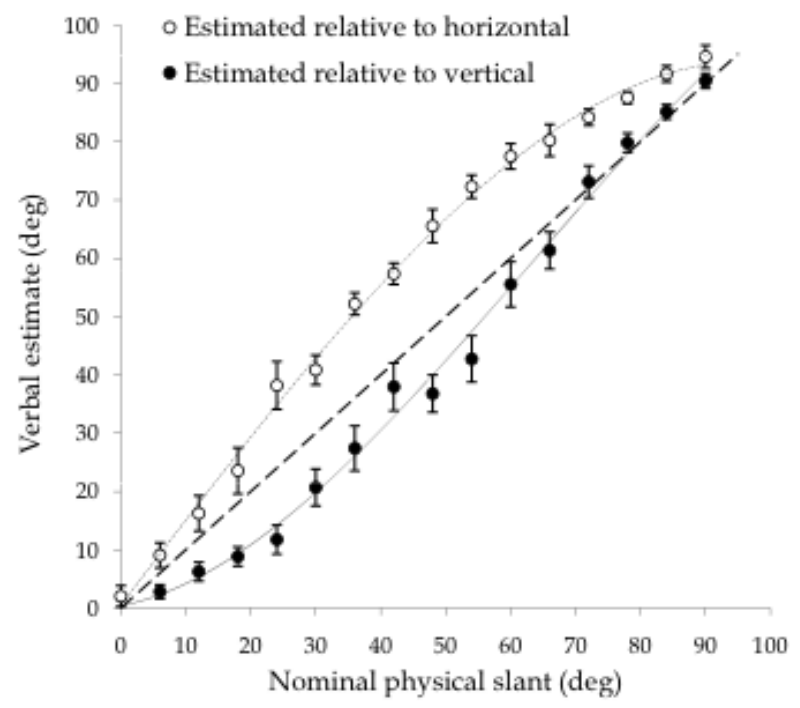

Fig. 9. Mean haptic slant estimates by reference condition in Experiment 1 with error bars representing \pm 1 SEM. Trend lines are best fitting cubic polynomials.

\subsection{Results}

Mean estimates were computed for each presented orientation by condition. Figure 9 shows the estimates for each condition. It can be seen that the spatial bias was in the same direction in each condition inasmuch as participants overestimated deviations from horizontal and underestimated deviations from vertical.

To represent the spatial bias function, we recalculated each estimate in the vertical referent condition with respect to horizontal and then averaged all estimates with respect to horizontal. The resulting function is plotted in Figure 10, superimposed on the similarlyderived spatial function for visual slant perception from Durgin, Li and Hajnal (2010, Experiment 1), plotted earlier in Figure 1. The functions are strikingly similar, as predicted 
by the calibration hypothesis. Both functions seem to reflect a common underlying spatial coding bias.

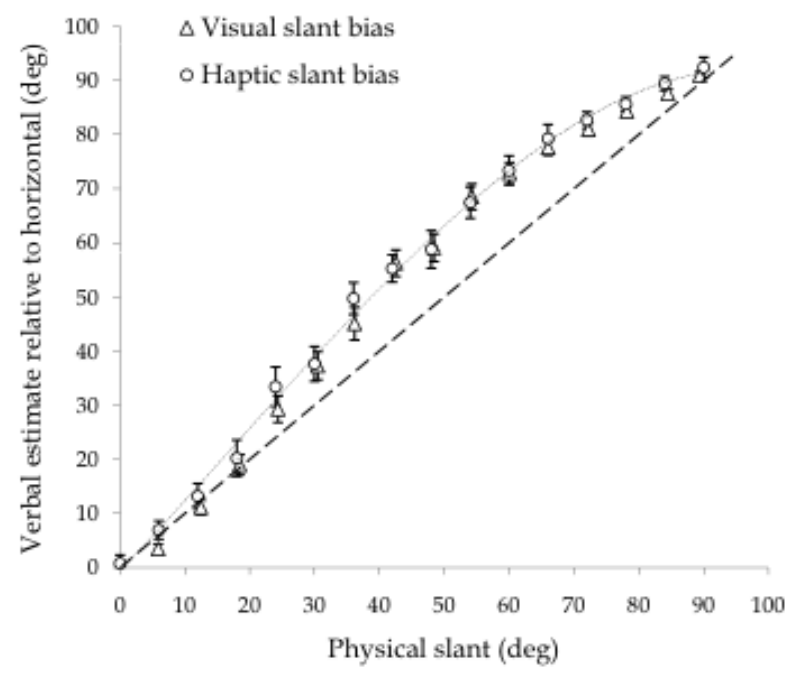

Fig. 10. Overall spatial bias in haptic perception of slant (Experiment 1) superimposed on the visual slant bias function from Durgin, Li and Hajnal (2010).

\subsection{Discussion}

Using real surfaces with a demarcated axis of haptic exploration, we sought to extend the methods used by Durgin, Li and Hajnal (2010) to the haptic domain. Our results indicate a close correspondence between visual and haptic spatial biases in the peception of orientation. Our results are somewhat at variance with those of Hajnal et al. (2011). Because Hajnal et al. did not constrain the path of digital exploration, it is possible that participants tended to explore their surfaces along a somewhat oblique (and therefore less steep) axis. Our data are consistent with the proposal that there is a trend for there to be calibration between visual and haptic representations of 3D surface orientation.

\section{Experiment 2: Horizontal/vertical bisection point for surface orientation in depth assessed by dynamic touch with the tip of the index finger}

To avoid verbal biases, Durgin, Li and Hajnal (2010) used a bisection task in which they presented surface visually and asked participants to indicate whether the surface was closer to vertical or to horizontal. They reported a mean visual bisection of point of $34^{\circ}$ from horizontal. That is, a surface of $34^{\circ}$ was equally likely to be judged closer to vertical as it was to be judged closer to horizontal. In fact the cubic fit to their verbal data predicted that the $45^{\circ}$ point would have been at $36.3^{\circ}$ in the visual case, and it seems likely that verbal reports tend to slightly underestimate the magnitude of the actual spatial bias (see also Durgin \& Li, 2011a, 2011b). The present experiment simply replicated the bisection procedure of Durgin, $\mathrm{Li}$ and Hajnal for the haptic case. 


\subsection{Method}

The haptic horizontal/vertical bisection point was measured.

\subsubsection{Participants}

The participants were 12 Swarthmore College undergraduate students who either participated in partial fulfilment of a course requirement or were paid to participate.

\subsubsection{Apparatus}

The apparatus was the same as in Experiment 1, except that a computer program was used to dynamically choose stimuli for presentation based on a staircase procedure designed to sample densely in the range surrounding each participant's subjective bisection point.

\subsubsection{Design}

Each participant gave responses to individual stimuli selected from an up-down staircase procedure. There were 10 blocks of 6 trials each in which two trials from each of three staircases were randomly interleaved. The starting values for the three staircases were either approximately $12^{\circ}, 42^{\circ}$, and $72^{\circ}(\mathrm{N}=6)$ or $18^{\circ}, 48^{\circ}$ and $78^{\circ}(\mathrm{N}=6)$. The step size of each staircase was $18^{\circ}$. That is, if the presented orientation was deemed closer to vertical, the next orientation presented by that staircase was $18^{\circ}$ lower, and if the presented orientation was judged closer to horizontal, the next presented orientation was $18^{\circ}$ higher. The three staircases together sampled orientation space with a resolution of $6^{\circ}$ and approximated a method of constant stimuli that was centered on the apparent bisection point.

\subsubsection{Procedure}

Participants were shown the apparatus with the surface in the horizontal position and the procedure was explained to them prior to signing an informed consent form. Participants were shown where to stand (directly in front of the apparatus) and then asked put on the blindfold. Before each trial, the surfaces were set to the required orientation manually by the experimenter according to a computer instruction. The participant then explored the surface as in Experiment 1. When the participant gave the forced choice response ("closer to vertical" or "closer to horizontal"), the experimenter pressed either the up-arrow key or the down arrow key on a keyboard, causing the computer to record the trial and update the staircase. The computer then gave instruction to the experimenter concerning the orientation of the next stimulus.

\subsection{Results}

The responses for each participant were fitted with a logistic function and the subjective bisection point was calculated for each psychometric function as the point at which participants were equally likely to respond that the surface was closer to vertical and that it was closer to horizontal. The average subjective horizontal/vertical bisection point was $31.2^{\circ}\left(\mathrm{SEM}=2.0^{\circ}\right)$ from horizontal. Although numerically lower than the $34^{\circ}$ average reported for visual slant by Durgin, Li and Hajnal (2010), this difference was not statistically reliable. The subjective bisection point did not differ reliably from $30^{\circ}, t(11)<1$, but did differ reliably from $45^{\circ}, t(11)=6.89, p<.0001$. 


\subsection{Discussion}

Using real surfaces with a clearly-demarcated axis of haptic exploration, we sought to extend the bisection method used by Durgin, Li and Hajnal (2010) to the haptic domain. Our results are consistent with a close correspondence between visual and haptic spatial biases in the perception of orientation. The haptic results are also quite similar to the average perceived horizontal/vertical bisection point $\left(31^{\circ}\right)$ measured by Durgin and Li (2011a) for perceived gaze declination. In other words, across a variety of modalities (proprioception of gaze declination, visual perception of 3D surface orientation in depth and haptic perception of 3D surface orientation in depth) the perceived bisection point between horizontal and vertical is very close to $30^{\circ}$ from horizontal.

\section{A descriptive model of the slant bias function for manual reaching space}

The main purpose of our present study has been to clear up an apparent discrepancy in the coding of near-space orientation. That is, for manual reaching space, there had seemed to be a discrepancy between the dynamic touch results of Hajnal et al. (2011) and the static haptic results of Durgin, Li and Hajnal (2010). The present results support the idea that the same bias exists for dynamic touch as has been found for visual slant perception and static haptic slant perception. The bias function found previously for the evaluation of visual slants and for haptic slants experienced by static contact has now been replicated for dynamic touch by fingertip. In combination with recent evidence that the proprioceptively-perceived orientation of a freely-extended hand shows a very similar bias function ( $\mathrm{Li} \&$ Durgin, submitted), the present results seem to point to a stable and systematic bias in the perception of $3 \mathrm{D}$ orientation in reachable space.

\subsection{A family of biases}

Our description is intentionally limited to 3D slant in manual reaching space because it is fairly clear that the underfoot haptic perception of ramp orientation, for example, follows a rather steeper function than the one for manually reachable surfaces (Hajnal et al., 2011). The shape of that function has only been explored over a limited range, however. Similarly, the bias function for the perceived orientation of palm boards (Bhalla \& Proffitt, 1999) has been shown to differ (especially at steeper orientations) from the haptic perception of stable surfaces (Durgin, Li \& Hajnal, 2010). There seems to be some continuity between the nearspace bias function and biases shown in hill perception, once distance is taken into account. That is, Li and Durgin (2010) argue that there is a perceptual gain of about 1.5 in the lower range of slants at all distances they tested, but a shift in the overall intercept. Durgin and Li (2011a) have argued that the 1.5 gain also applies to perceived gaze declination in the relevant range of declinations (i.e. out to about $45^{\circ}$ ).

With so many similar bias functions developed by recording verbal estimates of slant it is natural to wonder whether the bias might actually be in the numeric production system, but three important facts argue against this. First, non-numeric horizontal/vertical bisection tasks seem to provide converging evidence that a $3 \mathrm{D}$ slant or orientation of about $30^{\circ}$ appears to be equidistant between horizontal and vertical. This is consistent with the gain of 1.5 for the low range of angles. Second, in the present study, the bias function was very similar in shape when a very different set of numbers was required to produce it as a result 
of labelling vertical as $0^{\circ}$. Finally, the third important fact that argues against a purely numeric bias is that a very different bias function emerges when $2 \mathrm{D}$ orientation (of lines on a plane) is studied using similar numeric methods (Durgin and Li, 2011b). In this case, there is still a bias function that exaggerates deviations from horizontal, but absolute signed error peaks at $30^{\circ}$ rather than at $60^{\circ}$ as for the $3 \mathrm{D}$ function.

\subsection{Sine function scaling predicts the gain of $\mathbf{1 . 5}$}

The characteristic shape of the error functions we have observed somewhat resembles the first quarter cycle of a sine function. Such a function is plotted in Figure 11, scaled to $90^{\circ}$. Here we will first consider the features of the sine function that render it a promising model.

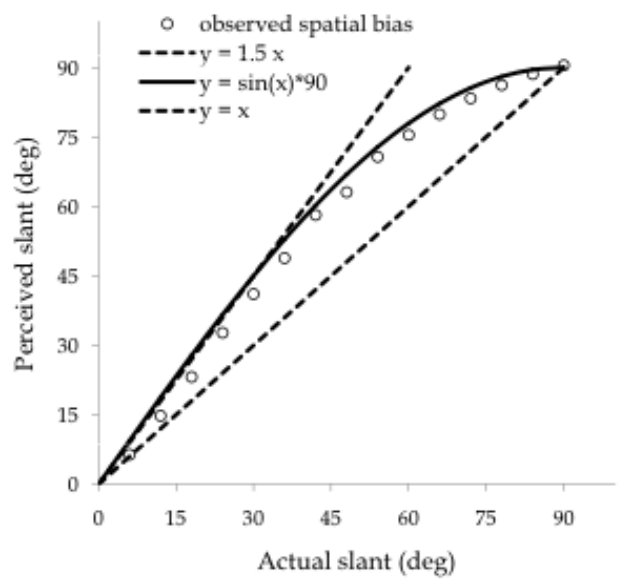

Fig. 11. Sine of slant, scaled to $90^{\circ}$. The sine function has a gain of essentially 1.5 over the range from 0 to nearly $45^{\circ}$. Means of pooled visual and haptic data are shown.

There are two features of particular note in Figure 11. First, the sine function captures the main shape of the bias functions we have been discussing: It appears fairly linear at the low end of the scale and compressive at the high end. The second point is made graphically by the line in Figure 11 representing a gain of 1.5 from horizontal. It turns out that the sine function produces a bias function with a gain of essentially 1.5 at the low end of the scale. Given the variety of empirically observed angular bias functions that have proven to have nearly exactly that gain near horizontal (e.g., visually perceived optical slant, haptically and visually perceived geographical slant, perceived gaze declination), this seems like either a striking coincidence or an impressive quantitative match.

A sine function represents the ratio between the surface length and the vertical extent of the surface. Unlike grade, which corresponds to the tangent function (rise over run), the sine function would seem to place priority on surface length, which has the virtue of being an ecologically relevant variable. For perceived gaze declination along a ground plane, where the relevant vertical extent is normally the observer's eye height, the sine of gaze declination corresponds to the reciprocal of the optical distance from the observer's eye to the point at the center of regard along the ground. If the optical distance from the eye to a target is held fixed, then the sine function is proportional to the frontal vertical extent created between the 
target and the horizontal plane at eye level. It remains unclear why these specific ratios might be used as a scale of angle. It is possible that the sine function only incidentally matches the underlying bias function. Durgin and Li (2011a; see also Hajnal et al., 2011; Li \& Durgin, 2009) have proposed that the shape of the bias function is driven by the utility of expanding the portion of the range used most frequently. At present we only propose that the sine function appears to capture the shape of the 3D angular bias function remarkably well.

\subsection{An implied direction of calibration?}

The idea that haptic scaling of perceived orientation during dynamic touch might be based on the ratio between the vertical extent of (finger-tip) travel and the total extent of (fingertip) travel is intriguing. Gravitational forces are highly relevant to haptics. The coding of orientation in this form would serve to express a useful ratio. When there is no vertical movement, the surface is horizontal. When the vertical component equals the total extent of travel, the surface is vertical. When the vertical extent is half the total extent of travel (i.e., $\left.\sin \left(30^{\circ}\right)=0.5\right)$, the surface is "half-way" between horizontal and vertical (i.e., the perceived bisection point). These facts fit our data well and seem appropriate for manual haptics. It is much less clear why the visual perception of the slant of manually-reachable surfaces should be similarly coded, given that neither the vertical extent of orientation change nor the overall surface extent are directly given in vision. However, if vision is calibrated to haptic experience, this would be sufficient to suggest why the visual and haptic codings are aligned, but also why the haptic experience of individuals who are congenitally blind are distorted in a similar manner. Insofar as it depends on manual haptic exploration, this hypothesis would apply specifically to manually-reachable surfaces. An implied direction of calibration does not require that calibration always go in this direction, but only supposes that there is a natural basis for sinusoidal scaling of manual haptic slant perception and that this basis could then drive the visual scaling.

\subsection{Scale expansion theory}

For large scale (locomotor) space, Durgin and Li (2011a) have proposed that the special role of proprioception of gaze direction in estimating distance (e.g., Wallach \& O'Leary, 1982) may encourage scale expansion near horizontal with a gain of 1.5. Because their model provides impressive quantitative predictions of perceptual matching tasks (Li, Phillips \& Durgin, 2011), it seems to capture an important feature of locomotor space perception. Durgin and Li have proposed that the 1.5 gain in the scaling of perceived slant may be driven by the 1.5 gain in gaze proprioception. That is, for horizontal ground surfaces to look flat requires a 1.5 gain in the optical slant. Thus, it might be argued that the expanded scale of perceived gaze declination also creates pressure for an expanded scale of visual slant.

\section{Conclusion}

In this chapter we reviewed basic knowledge concerning spatial biases in the perception of slant and then presented novel experimental results. Our experiments tested whether the perceived 3D orientation bias function for surfaces explored by dynamic touch was similar to that for visually perceived slant and static haptic touch. We found evidence that supports the view that the spatial bias for the perceived 3D orientation of surfaces in manual reaching 
space is similar across visual and haptic modalities whether measured numerically with respect to vertical or with respect to horizontal or even when measured non-verbally using a horizontal-vertical bisection task. We have further suggested that the orientation bias function in manual reaching space resembles the first quarter cycle of a sine function.

\section{Acknowledgments}

This research was supported by Award Number R15 EY021026-01 from the National Eye Institute. The content is solely the responsibility of the authors and does not necessarily represent the official views of the National Eye Institute or the National Institutes of Health. This work was also supported by a Swarthmore College Faculty Research Grant.

\section{References}

Bhalla, M. \& Proffitt, R.D. (1999). Visual-motor recalibration in geographical slant perception. Journal of Experimental Psychology: Human Perception and Performance, Vol. 25, No. 4, pp. 1076-1096

Bridgeman, B. \& Hoover, M. (2008). Processing spatial layout by perception and sensorimotor interaction. Quarterly Journal of Experimental Psychology, Vol. 61, pp. 851-859, Available from doi: 10.1080/1747021070162371

Durgin, F. H.; Baird, J. A.; Greenburg, M.; Russell, R.; Shaughnessy, K. \& Waymouth, S. (2009). Who is being deceived? The experimental demands of wearing a backpack. Psychonomic Bulletin \& Review, Vol. 16, No. 5, pp. 964-969, Available from doi: 10.3758/PBR.16.5.964

Durgin, F. H.; Hajnal, A.; Li, Z.; Tonge, N. \& Stigliani, A. (2010). Palm boards are not action measures: An alternative to the two-systems theory of geographical slant perception. Acta Psychologica, Vol. 134, No. 2, pp. 182-197, Available from http:/ / dx.doi.org/10.1016/j.actpsy.2010.01.009

Durgin, F. H.; Hajnal, A.; Li, Z.; Tonge, N. \& Stigliani, A. (2011). An imputed dissociation might be an artifact: Further evidence for the generalizability of the observations of Durgin et al. 2010. Acta Psychologica, Vol. 138, No. 2, pp. 281-284. Available from http://dx.doi.org/10.1016/j.actpsy.2010.09.002

Durgin, F. H. \& Li, Z. (2011a). Percepual scale expansion: An efficient angular coding strategy for locomotor space. Attention, Perception \& Psychophysics, Vol. 73, No. 6, pp. 1856-1870. Available from doi: 10.3758/s13414-011-0143-5

Durgin, F. H. \& Li, Z. (2011b). The perception of 2D orientation is categorically biased. Journal of Vision, Vol. 11, No. 8, Art. 13, pp. 1-10, Available from http://www.journalofvision.org/content/11/8/13

Durgin, F. H.; Li, Z. \& Hajnal, A. (2010). Slant perception in near space is categorically biased: Evidence for a vertical tendency. Attention, Perception $\mathcal{E}$ Psychophysics, Vol. 72, No. 7, pp. 1875-1889, Available from doi: 10.3758/ APP.72.7.1875

Feresin, C.; Agostini, T. \& Negrin-Saviolo, N. (1998). Testing the validity of the paddle method for the kinesthetic and visual-kinesthetic perception of inclination, BehaviorResearch Methods, Instruments \& Computers, Vol. 30, No. 4, pp. 637-642, Available from doi: 10.3758/BF03209481

Gibson, J. J. (1950). The perception of visual surfaces. The American Journal of Psychology, Vol. 63, No. 3, pp. 367-384 
Hajnal, A.; Abdul-Malak, D. T. \& Durgin, F. H. (2011). The perceptual experience of slope by foot and by finger. Journal of Experimental Psychology: Human Perception and Performance, Vol. 37, No. 3, pp. 709-719. Available from doi: 10.1037/a0019950

Kammann, R. (1967). Overestimation of vertical distance and slope and its role in moon illusion. Perception \& Psychophysics, Vol. 2, No. 12, pp. 585-589, Available from doi: 10.3758/BF03210273

Kinsella-Shaw, J. M.; Shaw, B. \& Turvey, M. T. (1992). Perceiving “Walk-on-able” slopes. Ecological Psychology, Vol. 4, No., pp. 223-239.

Li, Z. \& Durgin, F. H. (2009). Downhill slopes look shallower from the edge. Journal of Vision, Vol. 9, No. 11, Art. 6, pp. 1-15. Available from http://journalofvision.org/9/11/6

Li, Z. \& Durgin, F. H. (2010). Perceived slant of binocularly viewed large-scale surfaces: A common model from explicit and implicit measures. Journal of Vision, Vol 10, No. 14, Art. 13, pp. 1-16. http://journalofvision.org/content/10/14/13

Li, Z. \& Durgin, F. H. (2011). Design, data and theory regarding a digital hand inclinometer: A portable device for studying slant perception. Behavior Research Methods, Vol. 43, No. 2, pp. 363-371, Available from doi: 10.3758/s13428-010-0047-7

Li, Z. \& Durgin, F. H. (submitted). Manual matching of perceived surface orientation is affected by arm posture: Evidence of calibration between hand action and visual experience in near space.

Li, Z.; Phillips, J. \& Durgin, F. H. (2011). The underestimation of egocentric distance: Evidence from frontal matching tasks. Attention, Perception \& Psychophysics, Vol. 73, No. 7, pp. 2205-2217, Available from doi: 10.3758/s13414-011-0170-2

Proffitt, D. R.; Bhalla, M.; Gossweiler, R. \& Midgett, J. (1995). Perceiving geographical slant. Psychonomic Bulletin \& Review, Vol. 2, No. 4, pp. 409-428

Ross, H. E. (1974). Behaviour and perception in strange environments, Allen \& Unwin, ISBN 0041500474, London

Wallach, H. \& O'Leary, A. (1982). Slope of regard as a distance cue. Perception $\mathcal{E}$ Psychophysics, Vol. 31, No. 2, pp. 145-148, Available from doi: 10.3758/BF03206214 


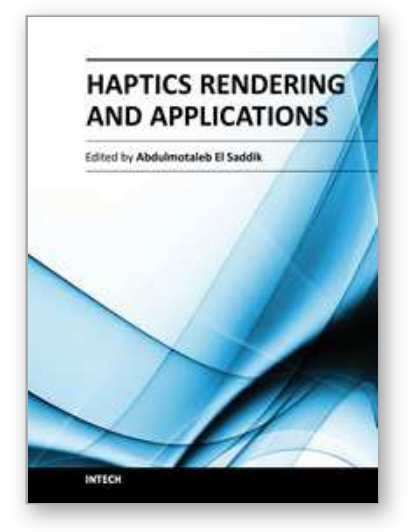

\author{
Haptics Rendering and Applications \\ Edited by Dr. Abdulmotaleb El Saddik
}

ISBN 978-953-307-897-7

Hard cover, 246 pages

Publisher InTech

Published online 27, January, 2012

Published in print edition January, 2012

There has been significant progress in haptic technologies but the incorporation of haptics into virtual environments is still in its infancy. A wide range of the new society's human activities including communication, education, art, entertainment, commerce and science would forever change if we learned how to capture, manipulate and reproduce haptic sensory stimuli that are nearly indistinguishable from reality. For the field to move forward, many commercial and technological barriers need to be overcome. By rendering how objects feel through haptic technology, we communicate information that might reflect a desire to speak a physicallybased language that has never been explored before. Due to constant improvement in haptics technology and increasing levels of research into and development of haptics-related algorithms, protocols and devices, there is a belief that haptics technology has a promising future.

\title{
How to reference
}

In order to correctly reference this scholarly work, feel free to copy and paste the following:

Frank H. Durgin and Zhi Li (2012). Spatial Biases and the Haptic Experience of Surface Orientation, Haptics Rendering and Applications, Dr. Abdulmotaleb El Saddik (Ed.), ISBN: 978-953-307-897-7, InTech, Available from: http://www.intechopen.com/books/haptics-rendering-and-applications/spatial-biases-and-the-hapticexperience-of-surface-orientation

\section{INTECH}

open science | open minds

\author{
InTech Europe \\ University Campus STeP Ri \\ Slavka Krautzeka 83/A \\ 51000 Rijeka, Croatia \\ Phone: +385 (51) 770447 \\ Fax: +385 (51) 686166 \\ www.intechopen.com
}

\author{
InTech China \\ Unit 405, Office Block, Hotel Equatorial Shanghai \\ No.65, Yan An Road (West), Shanghai, 200040, China \\ 中国上海市延安西路65号上海国际贵都大饭店办公楼 405 单元 \\ Phone: +86-21-62489820 \\ Fax: $+86-21-62489821$
}


(C) 2012 The Author(s). Licensee IntechOpen. This is an open access article distributed under the terms of the Creative Commons Attribution 3.0 License, which permits unrestricted use, distribution, and reproduction in any medium, provided the original work is properly cited. 